\title{
Die Bedeutung der Elektrokonvulsionstherapie (EKT) in der multimodalen Behandlung depressiver Störungen
}

\author{
Holger Himmighoffen \& Heinz Böker \\ Psychotherapie-Wissenschaft 10 (2) 61-73 2020 \\ www.psychotherapie-wissenschaft.info \\ CC BY-NC-ND \\ https://doi.org/10.30820/1664-9583-2020-2-61
}

\begin{abstract}
Zusammenfassung: Die Elektrokonvulsionstherapie (EKT) ist Teil des multimodalen Behandlungskonzepts in der Behandlung insbesondere depressiver Erkrankungen. Die therapieresistente Depression ist als ein wesentlicher Indikationsbereich anzusehen. Nach jahrzehntelanger Erfahrung erweist sich EKT weiterhin als sehr wirksame und aufgrund der heutigen Standards und Technik als sichere und relativ nebenwirkungsarme Behandlungsmethode. Daher überrascht ihre vorurteilsbeladene und negative Wahrnehmung und Bewertung. Auch die sehr häufige Einschätzung der EKT als eine «Ultima ratio» führt dazu, dass sie in vielen Fällen erst zu einem sehr späten Zeitpunkt im Krankheitsverlauf angewandt wird trotz anderslautender Empfehlung bspw. der S3-Leitlinie Unipolare Depression der DGPPN. Der Indikationsbereich für EKT ist klar umrissen und es gibt nur wenige absolute Kontraindikationen; auch eine Kombination von EKT mit anderen Behandlungsverfahren ist möglich und sinnvoll. Zentrales Anliegen dieses Artikels ist die notwendige Enttabuisierung: EKT sollte im Rahmen einer umfassenden Therapieplanung als mögliche Behandlungsoption angeboten werden. Oftmals ermöglicht erst sie die Überwindung depressiver Blockaden, die Auseinandersetzung mit biografisch relevanten Konfliktthemen, dysfunktionalen Bewältigungsmechanismen, traumatischen Erfahrungen und auch dem «existenziellen Thema der verlorenen Lebenszeit» infolge einer therapieresistenten Depression.
\end{abstract}

Schlüsselwörter: Elektrokonvulsionstherapie (EKT), therapieresistente Depression, Psychotherapie, multimodale Behandlung

\section{Geschichte und Grundlagen der EKT}

Die Elektrokonvulsionstherapie (EKT), früher auch als Elektrokrampftherapie und ursprünglich als «Elektroschocktherapie» bezeichnet, ist aktuell nach der Psychopharmakotherapie das wichtigste und am häufigsten eingesetzte somatische Behandlungsverfahren in der Psychiatrie (Sackeim, 2017). Sie wurde 1938 von den italienischen Psychiatern Ugo Cerletti und Lucio Bini entwickelt sowie erstmals angewendet und fand eine rasche internationale Verbreitung innerhalb der damaligen Psychiatrie, da zu dieser Zeit die meisten der heute verwendeten Psychopharmaka nicht existierten und EKT sich als besonders wirksam zur Behandlung verschiedenster psychischer Störungen (insbesondere schizophrene und depressive Erkrankungen) erwies (Reinke et al., 2013).

Das grundlegende Wirkprinzip der EKT ist die Auslösung eines kurzandauernden (30-60 Sekunden), generalisierten cerebralen Krampfanfalles mittels elektrischer Stimulation des Gehirns. Die Beobachtung, dass solche Krampfanfälle therapeutische Wirkungen haben, hatte man schon einige Jahre zuvor gemacht und bereits Krampfanfälle zu therapeutischen Zwecken bei psychisch Kranken ausgelöst. ${ }^{1}$

1 Zum Beispiel nutzte der ungarische Psychiater Ladislaus von Meduna Injektionen mit Kampfer (1934) und Cardiazol (1935) zur Anfallsauslösung.
Die EKT ist bei bestimmten Krankheitsbilden lebensrettend (z. B. bei perniziöser Katatonie) und wirksam bei einer grossen Zahl psychopathologischer Syndrome: Sie ist hoch wirksam antidepressiv, antimanisch, antisuizidal, antikataton und antikonvulsiv sowie teilweise auch antipsychotisch und stimmungsstabilisierend (Grager \& Di Pauli, 2013; Kellner, 2019). Trotzdem wurde und wird sie immer wieder kritisch beurteilt (vgl. z. B. die Übersichtsarbeiten von Read \& Bentall, 2010; Read \& Arnold, 2017), wobei dort der tatsächliche Effekt der EKT als zu gering im Verhältnis zum Ausmass der Nebenwirkungen gewertet wird.

Die EKT wurde sicherlich in den ersten Jahrzehnten nach ihrer Einführung 1938 sehr unkritisch und zu häufig und mit viel zu breiter Indikation ohne ausreichende Aufklärung und möglicherweise ohne Einverständnis der Patient"innen durchgeführt. Auch war die EKT bis circa Anfang der 1960er Jahre mit recht schweren Nebenwirkungen verbunden. So wurde bis dahin EKT ohne Narkose durchgeführt, das heisst im Wachzustand eingeleitet (der dann ausgelöste generalisierte cerebrale Krampfanfall ist mit einem Bewusstseinsverlust verbunden). Auch gab es bis Mitte der 1950er Jahre noch nicht die Möglichkeit einer sicheren und gut kontrollierbaren Muskelrelaxation, sodass die mit dem Krampfanfall verbundenen tonisch-klonischen Krämpfe der Körpermuskulatur nicht unterdrückt werden konnten. Dies führte 
durch die plötzlichen Kontrakturen der Muskulatur oft zu Muskel- und Sehnenverletzungen oder auch Knochenfrakturen (insbesondere der Wirbelsäule). Das erste verfügbare und heute weiterhin bei der EKT regelmässig eingesetzte Muskelrelaxans Suxamethonium (im Englischen auch als Succinylcholin bezeichnet) wurde in den 1950er-Jahren eingeführt und in der Schweiz 1954 zugelassen. Seit dem Einsatz von Muskelrelaxantien bei der EKT ist auch eine Narkose obligatorisch, da die bei wachen Patient*innen dann auftretende kurzzeitige Lähmung der Atemmuskulatur als extrem beängstigend erlebt wird. Auch waren kognitive Störungen wie postiktale Verwirrtheitszustände und Gedächtnisstörungen in den Anfangszeiten der EKT sehr viel häufiger und ausgeprägter, was mit der Intensität und der Art des verwendeten Stroms zur Anfallsauslösung zusammenhing. Ursprünglich wurde von Cerletti und Bini sinusförmiger Wechselstrom verwendet, im weiteren Verlauf der technischen Entwicklung der EKT-Geräte ab den 1960er Jahren dann kurz gepulster Gleichstrom (sog. Kurzpulstechnik) eingesetzt, wodurch die Menge abgegebener Ladung für die Auslösung eines Krampfanfalles reduziert und so das Ausmass der kognitiven Störungen verringert werden konnte.

Aufgrund der ab den 1960er Jahren laufenden Veränderungen und Weiterentwicklungen der EKT hinsichtlich Technik und Durchführung ist diese seitdem sicherer, weniger belastend und mit weniger Nebenwirkungen verbunden (sog. modifizierte EKT): Es gibt eine ausführliche mündliche und schriftliche Aufklärung (zumeist unter Einbeziehung Angehöriger) auf die ein schriftliches Einverständnis der Patient*innen für die Durchführung folgt (bei einer lebensbedrohlichen Notfallsituation und nicht bestehender Einwilligungsfähigkeit der Patient*innen wird die Durchführung aufgrund einer Patient*innenverfügung und Beiziehung von Vertretungspersonen wie Beistand, Ehepartner*in, Verwandten etc. geklärt). Die Durchführung der EKT erfolgt durch damit erfabrene Psychiater*innen in Kurznarkose (nach PräOxigenierung) und mit Muskelrelaxation (durchgeführt durch Anästhesist*innen). Vor, während und bis 2-3 Stunden nach der EKT-Sitzung findet ein Monitoring von Blutdruck, Herzfrequenz und Sauerstoff-Sättigung im Blut statt. Obligatorisch sind ausserdem eine EEG-Ableitung, fakultativ EMG (Elektromyografie) sowie der Einsatz von Gleichstrom, Rechteckimpulsen und Kurzpulstechnik. Bei erfolgreicher EKT-Serie (in der Regel 10-12 Sitzungen) wird ein Erhaltungs-EKT empfohlen.

Die modifizierte EKT ist heute anerkannter und international verbreiterter Standard bei der Behandlung. Auch wird die Indikation zu einer EKT heute präziser und enger gestellt. Doch vor dem Hintergrund der grossen Wirksamkeit, der verbesserten und sicheren Durchführung und der über viele Jahrzehnte bestehenden Erfahrungen mit der EKT ist es überraschend, wie stark vorurteilsbeladen und kritisch negativ EKT weiterhin in der Öffentlichkeit und auch von Fachpersonen wahrgenommen und bewertet wird und weniger häufig als eigentlich indiziert eingesetzt wird (Sackeim, 2017; Lauber et al., 2005; Grözinger et al., 2013). Auch die sehr häufige Einschätzung der EKT als eine «Ultima ratio» in der Behandlung von depressiven und anderen psychischen Störungen führt dazu, dass sie in vielen Fällen erst zu einem sehr späten Zeitpunkt im Verlauf einer Krankheitsepisode angewandt wird (vgl. Pfaff et al., 2013). Auch wird EKT - ganz zu Unrecht sehr oft mit Zwangsmassnahmen in der Psychiatrie in Verbindung gebracht.

In diesem Beitrag soll EKT als Behandlungsmethode mit ihren Wirkmechanismen und Wirkungen, ihren Indikationen und Kontraindikationen, ihren Risiken und Nebenwirkungen sowie ihrer Bedeutung innerhalb des multimodalen Behandlungskonzepts depressiver Störungen und ihrem Verhältnis zur Psychotherapie dargestellt werden.

\section{Wirkmechanismen der EKT}

Die Wirkung der EKT resultiert aus dem generalisierten cerebralen Krampfanfall und nicht aus dem elektrischen Strom, der appliziert wird. Neben dem Krampfanfall und möglicherweise auch der Suppression des Krampfanfalles als essenzielle Wirkmechanismen werden weitere diskutiert. So gibt es eine Reihe von Studien, die verschiedenste neurophysiologische und neurofunktionelle kurz- und langfristige Veränderungen durch EKT festgestellt haben:

> kurz- und langfristiger Anstieg neurotropher Faktoren wie BDNF (Brain Derived Nerve Factor; Okamoto et al., 2008; Piccinni et al., 2009; Rocha et al., 2016) und NGF (Nerve Growth Factor; Bilgen et al., 2014)

$>$ konsekutiv Neurogenese und Neuroplastizität im Hippocampus und anderen Hirnregionen (Bolwig \& Madsen, 2007; Lamont et al., 2005; Madsen, 2000; Michael et al., 2003a; Olesen et al., 2017; Otabe et al., 2014; Schloesser et al., 2015; Smith et al., 2014; Svensson et al., 2016; Tendolkar et al., 2013)

$>$ kurzfristig verstärkte Freisetzung von Neurotransmittern: Dopamin , Serotonin (Baldinger et al., 2014; Ishihara \& Sasa, 1999), GABA (Sanacora et al., 2003), Noradrenalin (Kelly \& Cooper, 1997)

> Beeinflussung der Rezeptorenfunktion im Gehirn: 5-HT3 , 5-HT1A , 5-HT2A - (Ishihara \& Sasa, 1999)

$>$ Freisetzung verschiedenster Hormone wie Prolaktin und TSH (Abrams \& Sukartz, 1985; Abrams, 1992; Aperia et al., 1985), Cortisol und ACTH (Fink \& Nemeroff, 1989; Florkowski et al., 1996), Endorphine (Abrams, 1992), Oxytocin (Scott et al., 1989; Whalley et al., 1987)

> Reduktion der frontalen kortikalen Hyperkonnektivität (bei depressiven Patient*innen) zwischen medialen Anteilen des Cortex (ACC und medialer frontaler Cortex), des DLPFC (dorsolateraler präfrontaler Cortex) beidseits und der links unilateralen Region im supramarginalen und angulären Gyrus und dem somatosensorischen Assoziationscortex (SAC) (Perrin et al., 2012)

> Normalisierung abnormer funktionaler Konnektivität (bei depressiven Patient*innen) bei zwei Netzwerkpaaren (posteriorer Default Mode/DMPFC und posterior Default Mode/linker DLPFC) (Abbott et al., 2013) 
$>$ Normalisierung bei depressiven Patient"innen im Vergleich zu psychisch Gesunden verminderter Glutamat/ Glutamin (Glx)-Konzentrationen im linken anterioren Cingulum (ACC) (Pfleiderer et al., 2003) und linken dorsolateralen präfrontalen Cortex (DLPFC) (Michael et al., 2003b)

Durch die Forschung mit neuen Untersuchungsmethoden bei Menschen in vivo sind neue Erkenntnisse betreffend der erwünschten und unerwünschten Effekte der EKT zu erwarten (z. B. mittels Single Spectron Emission Computed Tomography [SPECT], Positron Emission Tomography [PET], functional Magnetic Resonance Imaging [fMRI], Magnetic Resonance Spectroscopy [MRS], ComputerAssisted Quantitative Electroencephalography [QUEEG] oder EEG-Mapping/low resolution brain electro-magnetic tomography [LORETA]).

\section{Indikationen für eine EKT}

Die Hauptindikationen für EKT sind schwere depressive Episoden (bei unipolar depressiven und bipolaren Störungen), manische Episoden, schizophrene Störungen und katatone Zustandsbilder (Kellner, 2019) und EKT ist die Behandlungsoption erster Wahl, wenn schwere und oft lebensbedrohliche psychiatrische Symptome und Zustandsbilder vorliegen (wie akute Suizidalität, Malnutrition, schwere katatone oder psychotische Symptomatik).

In den Behandlungsempfehlungen der psychiatrischen Fachgesellschaften der Schweiz (SGAD, SGBP, SGPP) für die somatische Behandlung unipolar depressiver Störungen (Holsboer-Trachsler et al., 2016a, b) ist EKT indiziert bei schweren depressiven Episoden mit psychotischen Symptomen, depressiven Episoden mit Therapieresistenz oder in besonderen Situationen (wie z. B. schwere Suizidalität u. a.), die eine rasche Besserung erfordern. Dabei kann EKT zu jedem Zeitpunkt während der Behandlung erwogen werden und wird neben der Akutbehandlung auch für die Erhaltungstherapie empfohlen, insbesondere dann, wenn Patient"innen in der akuten Phase der Behandlung auf EKT angesprochen haben oder mehrere rezidivprophylaktische Therapieversuche mit Psychopharmaka nicht erfolgreich waren.

In der Behandlungsleitlinie der Deutschen Gesellschaft für Psychiatrie, Psychotherapie und Nervenheilkunde (DGPPN) wird als Hauptanwendungsbereich der EKT vor allem die therapieresistente Depression angegeben (DGPPN, 2017; DGBS \& DGPPN, 2020). Dort wird EKT als klar indiziert gesehen, wenn nach zwei Behandlungsversuchen mit Antidepressiva eine fehlende oder unzureichende Besserung der Symptomatik vorliegt. EKT ist dort die Behandlung erster Wahl bei hoher Dringlichkeit (z.B. bei vitaler Bedrohung, schwerer Suizidalität u.ä.), bei Kontraindikationen oder erhöhten Risiken für andere Behandlungen, bei Patient*innenwunsch oder auch anderen positiven prognostischen Faktoren für eine EKT (gute Erfahrungen bei früherer EKT, psychotische Symptome, psychomotorische Verlangsamung). Gleicher- massen ist EKT - gemäss der Behandlungsleitlinie der Deutschen Gesellschaft für Bipolare Störungen (DGBS) und DGPPN (DGBS \& DGPPN, 2020) - auch indiziert für die Behandlung depressiver Episoden bei einer bipolaren Störung (bipolare Depression). Die Indikationen für eine EKT sind in Tabelle 1 übersichtlich dargestellt.

\begin{tabular}{|c|c|}
\hline $\begin{array}{l}\text { Notfall- } \\
\text { indikationen }\end{array}$ & $\begin{array}{l}\text { perniziöse Katatonie } \\
\text { (unbehandelt hohe Letalität) } \\
\text { akute Suizidalität oder sonstige } \\
\text { lebensbedrohliche Zustände } \\
\text { (wie z.B. Nahrungs- und Trinkverweige- } \\
\text { rung) bei schweren affektiven, schizoaf- } \\
\text { fektiven oder schizophrenen Störungen } \\
\text { manisches Delir } \\
\text { therapierefraktäres malignes } \\
\text { neuroleptisches Syndrom }\end{array}$ \\
\hline $\begin{array}{l}\text { Therapie der } \\
\text { ersten Wahl }\end{array}$ & $\begin{array}{l}\text { schwere (unipolare oder bipolare) } \\
\text { Depression mit hoher Suizidalität, } \\
\text { Wahn und/oder ausgeprägtem Stupor } \\
\text { schizoaffektive Störung mit schwerer } \\
\text { Depression } \\
\text { bekanntes gutes Ansprechen auf EKT } \\
\text { zu grosses Risiko bei Anwendung an- } \\
\text { derer Behandlungen (insb. bei älteren } \\
\text { Patient*innen oder in der Schwanger- } \\
\text { schaft/Postpartum) } \\
\text { Patient*innenwunsch (bei gegebener } \\
\text { grundsätzlicher Indikation) }\end{array}$ \\
\hline $\begin{array}{l}\text { Therapie der } \\
\text { zweiten Wahl }\end{array}$ & $\begin{array}{l}\text { therapieresistente } \\
\text { (unipolare oder bipolare) Depression } \\
\text { therapieresistente, } \\
\text { nicht lebensbedrohliche Katatonien } \\
\text { therapieresistente Psychose, } \\
\text { Schizophrenie, schizoaffektive Störung } \\
\text { therapieresistente Manie } \\
\text { therapieresistente gemischte } \\
\text { affektive Episode }\end{array}$ \\
\hline
\end{tabular}

Tab. 1: Indikationen für eine Elektrokonvulsionstherapie

\section{Kontraindikationen für eine EKT}

Es gibt nur wenige absolute oder relative Kontraindikationen für eine Elektrokonvulsionstherapie. Grundsätzliche Voraussetzung für die Durchführung einer EKT ist eine Narkosefähigkeit. Ansonsten gibt es eine Reihe von Hirn-, Herz- und Gefässerkrankungen, die eine Kontraindikation darstellen, da sie aufgrund des kurzzeitigen Blutdruckanstiegs während des Krampfanfalles bei der EKT mit einem erhöhten Komplikationsrisiko vor allem für Blutungen einhergehen können (s. hierzu insb. Zeile 1 von Tab. 2). So gibt es zwar mehrere Fallberichte über komplikationsfreie Durchführungen bei sorgfältiger Blutdruckkontrolle (Mueller et al., 2009), aber auch einen Einzelfallbericht mit fatalem Ausgang nach Ruptur eines Bauchaortenaneurysmas (Dogan et al., 2016). Bei cerebralen Aneurysmen gibt es bisher einen Fallbericht einer Ruptur nach einer EKT (Gugger \& Dunn, 2019), 
in der gleichen Publikation aber auch Übersicht zu einer grösseren Fallzahl von Patient*innen mit vorbehandelten und nicht vorbehandelten Aneurysmen, bei denen die EKT komplikationsfrei verlief. Auch ein unzureichend eingestellter arterieller Hypertonus, eine koronare Herzkrankheit und ein erhöhter Hirndruck stellen relative Kontraindikationen und sollten vor Beginn einer EKT gut behandelt und eingestellt sein. Zeile 3 in Tabelle 2 nennt Umstände oder Erkrankungen, die keine Kontraindikation für eine EKT darstellen. Bei zusätzlichen komorbiden psychischen Störungen muss berücksichtigt werden, wie das Ausmass ist und ob aktuell andere spezifische Behandlungsmassnahmen nötig sind. Auch sollten Patient*innen darüber aufgeklärt werden, dass die komorbide Störung und deren spezifischen Symptome von der EKT nicht gebessert werden.

\begin{tabular}{|c|c|}
\hline $\begin{array}{l}\text { absolute } \\
\text { Kontra- } \\
\text { indikationen }\end{array}$ & $\begin{array}{l}\text { akuter Myokardinfarkt (<3 Monate) } \\
\text { akuter cerebraler hämorrhagischer oder } \\
\text { ischämischer Insult (<1 Monat) } \\
\text { rupturgefährdetes Aortenaneurysma } \\
<14 \text { Tage Clipping/Coiling eines cerebra- } \\
\text { len Aneurysmas } \\
\text { schwere kardiopulmonale Funktionsein- } \\
\text { schränkung (keine Narkosefähigkeit) } \\
\text { schwerer, nicht einstellbarer arterieller } \\
\text { Hypertonus } \\
\text { akuter Glaukomanfall* } \\
\text { erhöhter Hirndruck, Hirnödem* }\end{array}$ \\
\hline $\begin{array}{l}\text { relative Kontra- } \\
\text { indikationen }\end{array}$ & $\begin{array}{l}\text { koronare Herzkrankheit ohne schwere } \\
\text { Funktionseinschränkung* } \\
\text { cerebrales Aneurysma, cerebrale Gefäss- } \\
\text { missbildung* } \\
\text { schlecht eingestellter arterieller Hyper- } \\
\text { tonus* } \\
\text { intrazerebrale Raumforderung mit deut- } \\
\text { lichem Ödem* }\end{array}$ \\
\hline $\begin{array}{l}\text { keine Kontra- } \\
\text { indikation }\end{array}$ & $\begin{array}{l}\text { höheres Alter, Schwangerschaft, Herz- } \\
\text { schrittmacher, demenzielle Entwicklung } \\
\text { oder zusätzlich bestehende komorbide } \\
\text { andere psychische Störungen (wie } \\
\text { Persönlichkeits-, posttraumatische } \\
\text { Belastungs-, Ess-, Angst- und Zwangs-, } \\
\text { Schmerzstörungen, Abhängigkeitser- } \\
\text { krankungen u.a.) }\end{array}$ \\
\hline Iur & $\begin{array}{l}\text { n sind bei guter Vorabklärung (fach- } \\
\text { Id adäquater Behandlung und Einstel- } \\
\text { er EKT keine Kontraindikation mehr. }\end{array}$ \\
\hline
\end{tabular}

Tab. 2 Kontraindikationen für eine EKT

\section{Durchführung der EKT}

Im Rahmen der bereits erwähnten obligatorischen Aufklärung der Patient*innen (und ggf. Angehörigen) wird selbstverständlich auf die möglichen zu erwartenden Nebenwirkungen und insbesondere die kognitiven $\mathrm{Ne}$ benwirkungen eingegangen (am besten ergänzt durch
Erläuterungen von Erfahrungsbeispielen aus der Praxis), ehe ein schriftliches Einverständnis in die EKT (informed consent) erfolgt. Ausnahmesituationen wurden bereits genannt. Hier ist eine rasche Klärung auf Basis der in solchen Situationen vorgegeben gesetzlichen Regelungen angezeigt. Zusätzlich zur Aufklärung und Einverständniserklärung müssen vor dem Beginn der EKT einige somatische Routineabklärungen erfolgen: Wie vor jeder Narkose neben einer körperlichen Untersuchung ein Routinelabor und ein Elektrokardiogramm (EKG); dann eine Magnetresonanztomografie (MRI) des Kopfes (nicht älter als $1 \mathrm{Jahr}$ ) zum Ausschluss intracerebraler Veränderungen und gegebenenfalls eine Abdomen-Sonografie oder ein Röntgen-Thorax sowie bei relevanten somatischen Vorerkrankungen eine fachärztliche Kontrolle (s. Tab. 2).

Neben der klinischen psychischen Symptomatik und der Erhebung des psychopathologischen Befundes vor Beginn der EKT sollten quantitative Erhebungen der psychischen Symptomatik (z. B. bei Depression: BDI, HAMD, MADRS; bei Katatonie: Bush-Francis Catatonia Scale; bei Schizophrenie: PANSS) und des kognitiven Zustands (MMSE, MOCA) erfolgen. Diese werden dann eventuell im Verlauf, aber sicher nach Abschluss einer EKT-Serie und im Verlauf einer Erhaltungs-EKT sowie nach Abschluss der Gesamtbehandlung wiederholt durchgeführt. Eine EKT-Serie oder die Erhaltungs-EKT können grundsätzlich ambulant durchgeführt werden, wenn Patient*innen körperlich ausreichend stabil sind, selbstständig oder in Begleitung zu den Behandlungen kommen können (Absprachefähigkeit, Distanz, Transportfähigkeit) und eine gewisse Betreuung zu Hause durch Angehörige nach der Narkose und EKT gewährleistet ist. Bei Patient*innen, die durch die psychische Erkrankung zu stark eingeschränkt sind, die somatisch instabil sind oder als Risikopatient*innen gelten, sollte die EKT-Serie stationär erfolgen. Sollten Patient*innen vor einer ersten Behandlung sehr verunsichert wegen der Nebenwirkungen sein, kann diese zu Beginn stationär erfolgen und dann ambulant fortgesetzt werden.

Nach der Vorbereitung mit Anlegen eines regelmässigen Monitorings (Blutdruck, Herzfrequenz und SauerstoffSättigung) und Elektroden zur Ableitung der Hirnströme (EEG) und zur Stimulation, mit Legen eines intravenösen Zugangs und vorgängiger Sauerstoffgabe über eine Beatmungsmaske erfolgt eine Kurznarkose und Muskelrelaxation (durchgeführt durch Anästhesist*innen). Mittels eines Stromimpulses von einigen Sekunden Dauer, der über eine oder zwei Stimulus-Elektroden frontal oder temporal am Kopf appliziert wird, wird dann ein cerebraler Krampfanfall ausgelöst, der idealtypisch 30-60 Sekunden (EEG) dauert, hohe Amplituden sowie eine hohe Synchronizität zwischen beiden Hemisphären aufweist, von einer Sympathikus-Aktivierung (Herzfrequenzanstieg) begleitet wird und möglichst rasch und komplett spontan endet (Sartorius et al., 2013). Um einen Anfall auszulösen, der den geforderten Qualitätskriterien entspricht, gibt es einige technische und medizinische Variationsmöglichkeiten (Positionierung der Elektroden, Anpassung der Stimulationsparameter, Häufigkeit der Durchführung, 
Anpassung und Umstellung der eingesetzten Narkosemittel und zusätzlicher Medikamente u.a.m.), die man nutzen kann.

Eine einzelne EKT-Sitzung hat in der Akutbehandlung keinen ausreichenden klinischen Effekt, was bedeutet, dass eine Serie von Sitzungen erfolgen muss. Dabei werden insgesamt 10-12 Sitzungen geplant (die Anzahl kann je nach klinischem Verlauf und Krankheitsbild etwas variieren) und pro Woche 2-3 Sitzungen durchgeführt. Erste klinische Effekte sind in aller Regel nach der 5.-6. Sitzung spürbar (was aber auch sehr variieren kann).

Die meisten Medikamente, die die Patient*innen vor der EKT eingenommen haben, insbesondere Antidepressiva wie selektive Serotonin-Wiederaufnahmehemmer (SSRI), Antipsychotika und andere (für andere somatische Erkrankungen), können und sollen unverändert beibehalten werden. Die Einnahme von Medikamenten sollte wegen der Narkose (relative Nüchternheit) bis zu zwei Stunden vor der Behandlung mit wenig Wasser erfolgen. Es gibt einige Medikamente, deren Dosis reduziert werden muss oder die abgesetzt werden müssen:

$>$ Lithium (der Lithium-Spiegel sollte unter $0,4 \mathrm{mmol} / \mathrm{l}$ liegen)

$>$ Antiepileptika, Benzodiazepine, Barbiturate (absetzen oder auf geringe Dosis reduzieren; diese sind antikonvulsiv und erhöhen die Schwelle zur Auslösung eines Krampfanfalles, was die Durchfü hrung einer EKT verhindern kann)

$>$ tri- und tetrazyklische Antidepressiva, SNRI (Serotonin-Noradrenalin-Wiederaufnahmehemmer wie Venlafaxin und Duloxetin) (Dosis reduzieren oder evtl. absetzen, da das Risiko kardialer Arrhythmien durch diese erhöht ist)

$>$ bei Schizophrenien und Katatonien können Clozapin und andere Antipsychotika hingegen synergistisch mit der EKT wirken und das Ansprechen auf EKT verbessern (Schulz-Du Bois \& Conca, 2013)

Sollte eine EKT-Serie erfolgreich sein, das heisst eine (Teil-)Response oder eine Remission bewirken, dann ist in aller Regel im Anschluss dringend eine Fortsetzung der EKT (sog. Erhaltungs-EKT) und eine Psychopharmakotherapie (inkl. Re-Evaluation) empfohlen.

\section{Unerwünschte Wirkungen und Risiken von EKT}

Die EKT hat - wie jede medizinische Behandlung - unerwünschte Wirkungen und Risiken, die aber von den Patient*innen, der allgemeinen Öffentlichkeit und auch medizinischen Fachpersonen sehr häufig überschätzt und von Medien wie EKT-Gegner*innen und -Kritiker*innen deutlich überzeichnet werden. So gibt es einige vermeintliche der EKT zugeschriebene unerwünschte Wirkungen, die durch Studien klar widerlegt wurden:

Die Mortalität einer EKT-Behandlung liegt in grossen nationalen Kohorten unter 1:400000 Behandlungen und damit sehr deutlich unter der allgemeinen Mortalität bei Depressionen (Torring et al., 2017). Das Risiko, durch eine erfolgte EKT-Serie und sich anschliessende Erhaltungs-EKT später eine Demenz zu entwickeln, ist nicht erhöht (Chu et al., 2018; Osler et al., 2018). Das Risiko für Schlaganfälle ist durch eine EKT-Behandlung nicht erhöht, auch nicht nach vorangegangenem Schlaganfall (Rozing et al., 2019). EKT ruft keine nachweisbaren Hirnschädigungen hervor; bisherige Studien zeigen im Gegenteil sogar Hirnvolumenzunahmen in bestimmten Regionen (Nuninga et al., 2019; Sartorius et al., 2019; Takamiya et al., 2019; zwei Metaanalysen: Gbyl \& Videbech, 2018; Takamiya et al., 2018). Es gibt keinen klaren Hinweis auf ein erhöhtes Risiko nach einer EKT an einer Epilepsie zu erkranken (Bøg et al., 2018).

Die meisten tatsächlichen unerwünschten Wirkungen treten während der EKT-Serie mit 2-3 Sitzungen pro Woche auf. Dabei zählen zu den häufigsten kurzfristigen unerwünschten Wirkungen (während oder direkt nach einer EKT-Sitzung): Kopf- und Muskelschmerzen, Benommenheit und kurze Verwirrtheit nach dem Anfall, Übelkeit, kurzzeitig erhöhter Blutdruck und ein beschleunigter Puls (Tachykardie) sowie eine Verlangsamung des Pulses (Bradykardie) postiktal (Häufigkeiten nach Benbow \& Crentsil, 2004; s. Tab. 3). Diese lassen sich bei Bedarf gut symptomatisch behandeln (Schmerzmittel, Medikamente gegen Übelkeit, hohen Blutdruck u. a.) und klingen sehr rasch nach der EKT-Sitzung wieder ab.

\begin{tabular}{|l|r|r|r|}
\hline $\begin{array}{c}\text { Nebenwirkung } \\
(\%)\end{array}$ & \multicolumn{1}{|c|}{$\begin{array}{c}\text { schwer } \\
(\%)\end{array}$} & $\begin{array}{c}\text { mild/mo- } \\
\text { derat (\%) }\end{array}$ & \multicolumn{1}{|c|}{$\begin{array}{c}\text { gesamt } \\
(\%)\end{array}$} \\
\hline $\begin{array}{l}\text { Gedächtnis- } \\
\text { störungen }\end{array}$ & 10 & 62 & 72 \\
\hline Kopfschmerzen & 11 & 44 & 55 \\
\hline Schwindel & 10 & 52 & 62 \\
\hline Verwirrung & 3 & 41 & 44 \\
\hline Erbrechen & 0 & 9 & 9 \\
\hline andere* & 7 & 11 & 18 \\
\hline $\begin{array}{l}\text { * Muskelschmerzen, Inkontinenz, trockener Mund, Palpita- } \\
\text { tionen, Schwächegefühl, Übelkeit }\end{array}$ \\
\hline
\end{tabular}

Tab. 3: Anteil Patient*innen (in $\%, n=70$ ), die über milde/moderate und schwere Nebenwirkungen durch EKT berichteten

Seltene kurzfristige unerwünschte Wirkungen, die schwerwiegender sind, sind nach dem Krampfanfall auftretende (postiktale) stärkere Verwirrtheits- und Agitationszustände, Zahn- oder Zungenverletzungen, Herzrhythmusstörungen (kardiale Arrhythmien) und eine Asystolie, prolongierte Anfälle und im Rahmen der Narkose ein Larynxspasmus oder eine Aspiration.

Neben der kurzzeitigen Verwirrtheit, den kurzen Gedächtnis- und Orientierungsstörungen direkt nach einer EKT-Sitzung und dem Krampfanfall treten mittelfristig im 
Verlauf einer EKT-Serie sehr häufig temporäre kognitive Störungen auf, die insbesondere das Gedächtnis und selten die Orientierung oder auch exekutive Funktionen betreffen. Dabei finden sich folgende kognitive Störungen: Sehr häufig sind anterograde Amnesien (Gedächtnisstörungen ab Beginn der EKT-Serie, wobei neue Informationen nicht abgespeichert werden und später deswegen auch nicht erinnert werden können). Weniger häufig sind retrograde Amnesien (Gedächtnisstörungen kürzlich zurückliegender Ereignisse vor Beginn der EKT). Selten sind retrograde Amnesien, die das autobiografische Gedächtnis und Langzeitgedächtnis betreffen (gestörte Erinnerung deutlich länger, das heisst Jahre zurückliegender Ereignisse, die in der Regel nur durch eine Erfassung des autobiografischen Gedächtnisses feststellbar sind). Ebenfalls selten sind Orientierungsstörungen (räumlich und zeitlich) und Störungen einzelner exekutiver Funktionen (z. B. Störungen der Handlungsplanung und -ausführung).

Patient*innen sollten über kognitive Störungen, mit denen bei einer EKT-Serie gerechnet werden muss, eingehend informiert werden, und in dieser Zeit von wichtigen Entscheidungen ohne Unterstützung durch Angehörige und Vertrauenspersonen (wie z.B. Vertragsabschlüsse u. a.) Abstand nehmen. Die kognitiven Störungen durch die EKT müssen von bereits vor der EKT bestehenden und durch die Grunderkrankung bedingten oft sehr ausgeprägten kognitiven Störungen abgegrenzt werden. Wenn zusätzliche kognitive Störungen im Rahmen einer EKTSerie auftreten, sind diese zumeist so ausgeprägt, dass in dieser Zeit in der Regel keine Arbeitsfähigkeit gegeben ist. Die durch die EKT verursachten kognitiven Störungen bestehen nur temporär und bilden sich in der Regel innerhalb eines Zeitraums von 4-8 Wochen nach Abschluss einer EKT-Serie spürbar zurück (Kalisova et al., 2018; Semskova et al., 2010) und sind nach 6 Monaten bei den meisten Patient"innen nicht mehr nachweisbar (Nuninga et al., 2018); dies trifft auch für ältere Patient*innen zu (Obbels et al., 2018). Einzelne Patient"innen berichten über persistierende kognitive Störungen, insbesondere retrograde Amnesien, die zum Teil mit verbleibenden depressiven Symptomen und nicht mit objektiv messbaren Störungen korreliert sind und dann nicht klar von durch die Depression bedingten kognitiven Störungen abgegrenzt werden können (Fernie et al., 2014; Fraser et al., 2008). Nach Ende einer EKT-Serie sollte die davor bestehende kognitive Leistungsfähigkeit innerhalb von Wochen bis wenigen Monaten wieder erreicht werden. Eine Erhaltungs-EKT hat in aller Regel keine anhaltenden negativen Auswirkungen auf die Kognition.

\section{Herausforderungen in der Behandlung von Depressionen und die Bedeutung der EKT}

Depressionen sind sehr häufige Erkrankungen, das heisst, man kann davon ausgehen, dass im Laufe eines Jahres circa $7-12 \%$ der Bevölkerung und im Verlauf ihres Lebens circa $20 \%$ aller Menschen an einer Depression erkranken. Ein erster Erkrankungsgipfel bei unipolaren
Depressionen liegt zwischen dem 30. und 40. Lebensjahr, ein zweiter zwischen dem 50. und 60. Frauen sind von einer Depression zwei- bis dreimal häufiger betroffen als Männer und etwa die Hälfte aller Depressionen bleibt unbehandelt. Nach einer ersten schweren depressiven Episode besteht im ersten Jahr nach einer wirksamen Behandlung eine Rückfallwahrscheinlichkeit von 21-22\% (Keller et al., 1983; Kanai et al., 2003), nach 2-5 Jahren eine von $30 \%$ bzw. $42 \%$ (ebd.) und bezogen auf die Lebenszeit besteht nach einer ersten depressiven Episode eine sehr hohe Rückfallwahrscheinlichkeit von $80 \%$ (APA, 1994). Bei 20-40 \% der depressiv Erkrankten nimmt die Erkrankung einen chronischen Verlauf, das heisst diese sprechen nicht oder zu wenig auf Psychopharmakotherapie an (Kocsis, 2000). Auch besteht bei depressiv Erkrankten ein deutlich erhöhtes Suizidrisiko im Vergleich zur Allgemeinbevölkerung: Das Lebenszeitrisiko beträgt $10-15 \%$ der depressiv Erkrankten gegenüber 0,7\% in der Allgemeinbevölkerung (Baldessarini et al., 2003).

Vor diesem Hintergrund ist klar, dass ein grosser Bedarf an wirksamen Therapien für Depressionen besteht und dass insbesondere bei unzureichender Wirksamkeit der am häufigsten eingesetzten antidepressiven Behandlungsmassnahmen (Psychotherapie und Psychopharmakotherapie) weitere Behandlungsmöglichkeiten vonnöten sind. In dieser Hinsicht nimmt EKT als sehr wirksames, gut anwendbares und verträgliches Therapieverfahren eine wichtige Rolle ein und wird von den meisten psychiatrischen Fachgesellschaft empfohlen. Studien belegen die Wirksamkeit von EKT bei depressiven Störungen (UK ECT Review Group, 2003; Pagnin et al., 2004; Grager \& Di Pauli, 2013; Luccarelli et al., 2020), anderen psychiatrischen Krankheitsbildern und Syndromen wie Schizophrenien (Lally et al., 2016; Petrides et al., 2015) und bipolaren Störungen (Perugi et al., 2017; Bahji et al., 2019), Suizidalität (Kellner et al., 2005) und Katatonie (Luchini et al., 2015). Dabei ist die Wirksamkeit von EKT sehr hoch: Man kann davon ausgehen, dass bei Depressionen ohne Therapieresistenz (also ohne vorherige andere Behandlungsmassnahmen) oder schweren psychotischen Depressionen 80-90\% der Patient"innen auf die EKT ansprechen; im Falle von Depressionen mit Therapieresistenz (also mit vorherigen, nicht erfolgreichen anderen Behandlungsmassnahmen) sind dies etwa $50-70 \%$ (Milev et al., 2016; Grager \& Di Pauli, 2013). Nach einer Meta-Analyse von Haq et al. (2015) gelten als positive Prädiktoren für das Ansprechen einer Depression auf EKT eine vorgängige kurze Episodendauer, wenige Vormedikationsversuche, höheres Alter und psychotische Symptome, das heisst, die Wirksamkeit von EKT bei Depressionen nimmt vor allem mit längerer Dauer der Vorbehandlung ab. Ohne Einfluss auf das Ansprechen können gelten: Geschlecht, Alter bei Beginn der depressiven Episode, Anzahl Episoden, Symptomschwere, unipolare versus bipolare Depression und auch psychiatrischen Komorbiditäten. Das bedeutet, dass bei zusätzlich zu einer Depression bestehenden anderen psychischen Störungen keine Kontraindikation für eine EKT besteht (s. zuvor). Zwar kann bei diesen keine Besserung durch eine EKT 
erwartet werden, Patient*innen können sich aber durch die Besserung der Depressivität insgesamt besser fühlen und dann auch eher in der Lage sein, komorbide Störungen zum Beispiel psychotherapeutisch zu behandeln.

Sämtliche depressiven Symptome und Beschwerden können sich durch die EKT bessern, dabei sind deutliche Besserungen im Sinne einer Response, das heisst Reduktion des Ausprägungsgrades der depressiven Symptome vor Beginn der EKT um $50 \%$, sehr häufig und auch eine weitestgehende oder vollständige Rückbildung (Remission) keine Seltenheit. EKT ist zudem sehr wirksam gegen Suizidalität: Kellner et al. (2005) konnten in einer Untersuchung von mit EKT behandelten suizidalen Patient*innen zeigen, dass nach 1 Woche Behandlung bei $38 \%$, nach 2 Wochen bei $61 \%$ und nach Abschluss der EKT-Serie bei $81 \%$ der Patient*innen die Suizidalität komplett verschwunden war. Auch bestehende kognitive Störungen durch die Depression (Konzentration, Aufmerksamkeit) können sich bessern, was aber aufgrund der durch EKT verursachten temporären kognitiven Störungen (vor allem Gedächtnisstörungen) nicht wahrgenommen wird, sodass subjektiv und objektiv zunächst insgesamt eine kognitive Verschlechterung durch die EKT besteht, die aber spätestens nach 6 Monaten nicht mehr nachweisbar ist (Nuninga et al., 2018).

Studien haben auch gezeigt, dass bei einer erfolgreichen EKT-Serie ohne sich anschliessende Erhaltungs-EKT bei depressiven Störungen das Risiko eines Rückfalls deutlich höher ist als mit Erhaltungs-EKT (Sackeim et al., 2001; Kellner et al., 2006; Brown et al., 2014; Luccarelli et al., 2020). Die Erhaltungs-EKT erfolgt zunächst in einwöchentlichen Abständen, die dann im Verlauf schrittweise vergrössert werden (bis zu vierwöchentlichen Abständen). So kann man dann zum Beispiel nach 6 Monaten weiterer Behandlung mit circa 10 Erhaltungs-EKT-Sitzungen und Evaluation des Verlaufs über das weitere Vorgehen entscheiden (Abschluss der EKT oder doch weitere Fortsetzung in gleichen oder weiter grösser werdenden Abständen). Auch ist eine Re-Evaluation der Psychopharmakotherapie sinnvoll, da viele Patient*innen von einer zusätzlichen medikamentösen Behandlung profitieren, selbst wenn vor der EKT Psychopharmaka nicht wirksam waren (Sackeim et al., 2001; Brown et al., 2014).

Trotz der nachgewiesen Wirksamkeit und recht guten Verträglichkeit der EKT bei depressiven Störungen dauert es sehr oft sehr lange bis es zu einer Zuweisung zur EKT und Behandlung kommt: So wurde in einer Studie der Psychiatrischen Universitätsklinik (PUK) Zürich und der Psychiatrischen Klinik Königsfelden (Pfaff et al., 2013) die Überweisungspraxis zur Behandlung von therapieresistenten Depressionen mit EKT untersucht. Das mittlere Alter der Stichprobe betrug 51 Jahre, 67,5\% der Patient" innen waren Frauen, 32,5 \% Männer. Die Dauer der aktuellen Episode betrug im Mittel 68 Wochen (!), die mittlere Krankheitsdauer 15,2 Jahre, die Behandlungsdauer 13 Jahre. Im Mittel befanden sich die angemeldeten Patient*innen bisher etwa viermal in stationärer psychiatrischer Behandlung. Bei den angemeldeten depressiv Erkrankten wurden im Mittel 5,7 unterschiedliche Antidepressiva eingesetzt (Streuung: 1-17!), Kombinationstherapien wurden in $86 \%$, Augmentationsstrategien in $87 \%$ der Fälle durchgeführt (Lithium 72\%, Antiepileptika 47\%, T3/T4 16\%, Neuroleptika $76 \%$ ). Die Antidepressiva waren in $61 \%-$ nach Arzneimittel-Kompendium - ausdosiert worden. Serum-Spiegelkontrollen waren allerdings nur bei $40 \%$ der Patient*innen erfolgt. $81 \%$ der Patient*innen waren neben der antidepressiv-medikamentösen Behandlung zuvor auch mit Psychotherapie behandelt worden (im Mittel etwa 7 Jahre). Bei etwa der Hälfte der überwiesenen Patient"innen lag eine Therapieresistenz $(n=21)$ vor. Bei den depressiv Erkrankten, die mit EKT behandelt wurden, ergab sich eine signifikante Besserung der Symptomatik (HAMD-Mittelwert bei Eintritt 35,8, bei Austritt 12,8; BDI-Mittelwert bei Eintritt 33, bei Austritt 16). Etwa $30 \%$ der mittels EKT behandelten Patient*innen waren bei Austritt vollständig remittiert. Hinweise auf erhebliche kognitive Nebenwirkungen der EKT fanden sich bei dieser Stichprobe nicht.

Zusammenfasst wurden im Untersuchungszeitraum in beide psychiatrische Kliniken (PUK Zürich, Psychiatrische Klinik Königsfelden) überwiegend schwerstkranke Patient*innen mit jahrelanger Krankheits- und Behandlungsdauer überwiesen. Die meisten waren mit einer Vielzahl von Antidepressiva vorbehandelt worden. Der Wunsch der Klärung der Indikation seitens der überweisenden Psychiater*innen bezog sich insbesondere auf Patient*innen mit chronischer Depression. So lässt sich schlussfolgern, dass die Zuweisungen zur EKT bei der untersuchten Stichprobe nicht den Kriterien von «Therapieresistenz» entsprachen, sondern die EKT offensichtlich als «letzte Wiese» in der Behandlung der therapieresistenten Depression aufgefasst wurde.

\section{EKT und Psychotherapie}

Wie sieht nun das Verhältnis von EKT zu psychotherapeutischen Behandlungsverfahren bei der Depression aus? Geht es hier um einen Widerspruch oder eine Ergänzung? Schliessen EKT und Psychotherapie einander aus? (Vgl. Conca et al., 2005). Ein Grund, beide Behandlungsmethoden in einem Widerspruch zu sehen, könnte darin liegen, dass zu wenig die multiple Ätiologie depressiver Störungen gesehen wird, bei denen psychosoziale und somatische Aspekte gleichermassen von grosser Relevanz sind. So kann es passieren, dass biologisch orientierte Psychiater*innen zu wenig die psychosozialen, nichtbiologischen Aspekte von Depressionen berücksichtigen und Psychotherapeut" innen die somatischen, biologischen Aspekte und Korrelate von Depressionen übersehen (vgl. Pandarakalam, 2018).

Es ist unumstritten, dass psychotherapeutische Verfahren in der Depressionsbehandlung sehr wirksam und von zentraler Bedeutung sind (Meister et al., 2018). Dabei ist Psychotherapie bei leichter bis mittelgradiger Ausprägung der depressiven Symptomatik gegenüber einer alleinigen Psychopharmakotherapie als gleichwertig anzusehen, bei 
schwerer depressiver Symptomatik ist die Kombination von Psychotherapie und Pharmakotherapie der alleinigen Behandlung mit Psychotherapie überlegen und somit die Behandlung der Wahl (ebd.; Thase et al., 1997).

Verschiedene Autor*innen haben sich mit der Frage der Kombination von Psychotherapie und Psychopharmaka beschäftigt und deren Vor- und Nachteile diskutiert (König, 1999; Kay, 2007; Himmighoffen, 2009). In Analogie dazu kann man die Vorteile und Nachteile der Kombination von Psychotherapie und somatischen Behandlungsverfahren im Allgemeinen (d.h. Psychopharmaka, EKT und andere somatische Verfahren), also auch die Kombination von Psychotherapie und EKT diskutieren. Dies ist insbesondere vor dem Hintergrund schwerer oder chronischer und therapieresistenter Depressionen von wichtiger Bedeutung (McIntyre et al., 2014; Pandarakalam, 2018), da hier der multimodale Behandlungsansatz mehr und mehr an Bedeutung gewinnt.

Mögliche Vorteile: Durch die Besserung bzw. Remission der depressiven Symptomatik durch die EKT sind Patient*innen wieder besser in der Lage, die Psychotherapie für sich zu nutzen und dabei thematisierte Aspekte zu bearbeiten und umzusetzen. Die Überwindung depressiver Blockaden und weiterer einschränkender depressiver Symptome (wie Gefühle von Hilflosigkeit und Sinnlosigkeit, Passivität etc.) durch die EKT ermöglicht es Patient*innen, wieder Zugang zur eigenen psychischen Innenwelt zu finden, und erleichtert damit die Auseinandersetzung mit biografisch relevanten Konfliktthemen, dysfunktionalen Bewältigungsmechanismen, traumatischen Erfahrungen und nicht zuletzt auch dem «existenziellen Thema der verlorenen Lebenszeit» infolge einer Depression. Auch können Patient*innen wieder eigene, depressionsmindernde Ressourcen nutzen, die zuvor durch die schwere depressive Symptomatik eingeschränkt waren. So können mittels der EKT nicht nur die Wahrscheinlichkeit, sondern auch die Geschwindigkeit und das Ausmass des Ansprechens auf Psychotherapie und deren Nachhaltigkeit erhöht werden. Nach einer EKT-Serie und bei Erhaltungs-EKT kann die zusätzliche Psychotherapie die Fähigkeiten zu Anpassung und Coping und die Akzeptanz einer somatischen Behandlung verbessern sowie das Risiko von Rückfällen vermindern (vgl. Brakemeier et al., 2014).

Mögliche Nachteile: Patient*innen könnten Verbesserungen ihres Befindens durch EKT ausschliesslich auf diese zurückführen und dann eine Psychotherapie als nicht erforderlich ansehen. So kann es passieren, dass depressionsfördernde Persönlichkeitsaspekte ausgeblendet und nicht bearbeitet werden bzw. antidepressive Eigenschaften und Ressourcen nicht aktiviert und gefördert werden. Eine daraus folgende negative Bewertung von Psychotherapie und die Idealisierung der "wirksamen" EKT durch Patient*innen können auch im Sinne einer Abwehr oder Verleugnung schwer erträglicher Gefühle und Gedanken verstanden werden, die sicherlich weitere Exploration und psychotherapeutische Bearbeitung erfordern würden. Ganz im Gegensatz dazu können Patient*innen sich durch den Einsatz von EKT von
Behandelnden «abgespeist» fühlen und den Eindruck gewinnen, dass diese ihre Gefühle und Gedanken nicht ertragen und nicht zum Thema machen möchten. Es ist sehr gut denkbar, dass Behandelnde ihre Patient"innen eine EKT vorschlagen, um sich selbst vor deren schwer aushaltbaren Gefühlen, Gedanken und Beschwerden zu schützen, denen sie in der therapeutischen Begegnung mit ihnen ausgesetzt sind. Die temporären kognitiven Störungen während einer EKT-Serie und in den ersten 1-2 Monaten danach (Gedächtnisstörungen) können für eine gewisse Zeit eine laufende intensive psychotherapeutische Arbeit erschweren, das heisst, es kann in dieser Zeit sinnvoll sein bspw. psychotherapeutisch mehr «ich-stützend» und begleitend vorzugehen.

Anhand von zwei Fallbeispielen möchten wir abschliessend die Wirkungen und Möglichkeiten von EKT bei depressiven Störungen und ihr Verhältnis zur Psychotherapie illustrieren. ${ }^{2}$

\section{Fallbeispiel 1}

Frau S., eine 69-jährige Patientin, die seit mehreren Jahrzehnten an einer bipolaren affektiven Störung leidet, wurde wegen einer schweren, therapieresistenten Depression in der PUK Zürich aufgenommen. Zuvor war sie vergeblich über zwei Jahre lang mit unterschiedlichen Kombinationen von Antidepressiva und stimmungsstabilisierenden Mitteln behandelt worden. Die erste schwere depressive Episode war nach Geburt des ersten Sohnes aufgetreten. In den folgenden Jahrzehnten traten - neben den Depressionen - zunehmend gehäuft manische Episoden mit ausgeprägter Agitation auf. Bei der stationären Aufnahme bestand ein depressiver Stupor, das heisst, die Patientin war völlig erstarrt und blockiert, war mutistisch und verweigerte Kontaktaufnahme und Nahrung. Ein weiterer medikamentöser Behandlungsversuch nach Klinikeintritt führte ebenfalls nicht zu einer Besserung. Daraufhin wurde der Patientin die Durchführung einer EKT empfohlen. Frau S. und ihr Ehemann wurden ausführlich informiert und willigten schliesslich ein.

Bereits nach 4 EKT-Sitzungen trat eine deutliche und zunehmende Besserung der Stimmung ein. Die psychomotorische Erstarrung der Patientin löste sich schrittweise. Frau S. konnte nach 12 EKT-Sitzungen nahezu beschwerdefrei nach Hause entlassen werden. Im Anschluss an die EKT-Serie wurde die Patientin erneut auf ein Antidepressivum eingestellt, ferner wurde ein Mood Stabilizer, den die Patientin auch in der Vergangenheit gut vertragen hatte, eingesetzt.

Im Verlauf der ambulanten Nachbetreuung wurde die Verabreichung der Psychopharmaka angepasst. Angesichts der Vorgeschichte mit zahlreichen depressiven und manischen Episoden und zunächst weiterhin bestehender Stimmungsschwankungen wurde eine ambulante Erhaltungs-EKT durchgeführt (zunächst wöchentlich, im weiteren Verlauf EKT-Sitzungen in vier- bis sechswö-

2 Alle personenbezogenen Angaben wurden anonymisiert. 
chentlichen Abständen). Die Stimmung der Patientin blieb schliesslich auch im längeren, mehrjährigen Verlauf stabil. Heute ist Frau S. weiterhin sehr unternehmungslustig, treibt Sport (was sie jahrelang während der depressiven Erkrankung nicht tun konnte) und unterhält vielfältige soziale Beziehungen innerhalb und ausserhalb ihrer Familie. Auch ihre Angehörigen sind sehr froh und entlastet. Sie nehmen eine - nach Jahren des Rückzugs und der Erstarrung - «unglaubliche, positive Veränderung» wahr. Kritisch fragen sie, warum Jahrzehnte vergehen mussten, bevor die Behandlung mittels EKT begonnen wurde. In der begleitenden Psychotherapie der Patientin und in den Paargesprächen mit ihrem Ehemann stellten der «Schmerz über die verlorene Lebenszeit» und die Ohnmacht des stets hilfreich-unterstützenden Ehemannes, der zunehmend erschöpft war, wesentliche Therapiefoki dar. Darüber hinaus konnten erstmals traumatische Erfahrungen der Patientin in ihrer durch den Krieg überschatteten Kindheit und im Zusammenhang mit erlebter emotionaler Deprivation bearbeitet werden.

\section{Fallbeispiel 2}

Herr C., 35 Jahre alt, verheiratet, zwei Kinder, wird ambulant wegen einer seit einem Jahr anhaltenden und therapieresistenten schweren depressiven Episode bei rezidivierender depressiver Störung zur Frage einer EKT zugewiesen. Eine erste depressive Episode hatte er im Alter von 20 Jahren und war danach 14 Jahre remittiert. Ein möglicher Auslöser der aktuellen depressiven Episode kann der unerwartete Tod des Schwagers nur einen Monat vor Beginn der depressiven Episode gewesen sein.

Im Verlauf des Jahres vor Zuweisung war eine dreimalige psychiatrische Hospitalisation mit jeweils einer gewissen Besserung durch die medikamentösen und psychotherapeutischen Behandlungen, die ambulant regelmässig fortgesetzt wurden, erfolgt. Trotz medikamentöser Mehrfachkombinationen und Augmentationsstrategien gab es jedoch auch immer wieder depressive Rückfälle bis hin zur Arbeitsunfähigkeit. Innerhalb seiner Familie fühlte sich Herr C. isoliert, erlebte sich unfähig, ein Teil der Familie zu sein, sich einzubringen und im lebendigen Kontakt mit der Ehefrau und seinen Kindern zu stehen, wie dies vor der Depression problemlos möglich gewesen war. Vor Beginn der EKT-Serie bestand ein schweres depressives Syndrom ohne psychotische Symptome, aber mit häufigen Suizidgedanken (ohne konkrete Handlungsansätze) und ohne Hoffnung auf Besserung.

Nach Durchführung einer ambulanten EKT-Serie mit insgesamt 12 Sitzungen innerhalb von 5 Wochen stellten sich eine deutlich positive Wirkung (Response) und sehr gute Verträglichkeit, das heisst kaum kognitive Störungen, ein. Sein Depressions-Rating vor Beginn der EKT-Serie war: BDI-II: 40 Punkte, HAMD-21: 27 Punkte, MMS 30/30 Punkte; und nach Abschluss der EKT-Serie: BDI-II: 14 Punkte, HAMD-21: 11 Punkte, MMS 30/30 Punkte. Es wurde eine medikamentöse Kombinationsbehandlung mit einem SNRI (Venlafaxin ER 112,5 mg/d) und
Lithium (Quilonorm Retard 1-0-1 Tabletten) fortgesetzt bzw. angepasst und Herr C. war neben der weiteren Erhaltungs-EKT wöchentlich in ambulanter psychiatrischpsychotherapeutischer Behandlung bei einer niedergelassenen Psychiaterin.

Zwar hinsichtlich der depressiven Symptomatik deutlich gebessert (deutliche Response), erlebt sich Herr C. zuerst jedoch weiterhin als unfähig, im lebendigen Kontakt mit der Ehefrau und seinen Kindern zu stehen. Seine Arbeit konnte er mit einem Pensum von $20 \%$ wieder aufnehmen und im weiteren Verlauf schrittweise langsam steigern. Im weiteren Verlauf erfolgte über einen Zeitraum von 11 Monaten eine Erhaltungs-EKT mit insgesamt 10 Sitzungen. Danach war eine weitere Besserung der Depression bis zur Remission festzustellen (BDI-II: 4 Punkte, HAMD-21: 1 Punkt).

Herr C. arbeitet nun wieder zu $100 \%$, ist psychisch stabil und hat keine affektiven Schwankungen mehr. Er fühle sich wieder so wie vor der Depression, erlebe sich wieder viel besser im Kontakt mit seiner Ehefrau und seinen Kindern. Auch äusserte er von sich aus, dass er in den letzten Monaten das, was in der weiterhin laufenden wöchentlichen Psychotherapie erörtert werde, nun für sich nutzen und umsetzen könne. Es wurden noch zwei weitere Erhaltungs-EKT-Sitzungen im Verlauf der folgenden 4 Monate durchgeführt. Danach erfolgte ein Verlaufsgespräch, indem aufgrund anhaltenden psychischen Wohlbefindens und konstanter psychischer Stabilität der Abschluss der EKT festgelegt wurde.

\section{Zusammenfassung und Ausblick}

EKT ist ein wichtiger Teil des multimodalen Behandlungskonzeptes in der Behandlung insbesondere depressiver Erkrankungen. Dabei ist die therapieresistente Depression als ein wesentlicher Indikationsbereich anzusehen. Nach jahrzehntelanger Erfahrung und Verbesserung der Durchführung und Technik seit den 1960er Jahren erweist sich die EKT als eine sehr wirksame, sichere und relativ nebenwirkungsarme Behandlungsmethode.

Bemerkenswert ist eine weiterhin oftmals negative Wahrnehmung dieser für eine Untergruppe depressiv Erkrankter sehr wichtigen Therapieoption in der Öffentlichkeit (Einer flog über das Kuckucksnest), zum Teil auch bei Vertreter*innen der Medizin und Psychiatrie, der Psychologie und Psychotherapie. Bei den zuletzt genannten Professionellen ist dies offensichtlich Folge der unzureichenden Verankerung des Themas und Auseinandersetzung mit der EKT in den jeweiligen beruflichen Weiterbildungen.

Die Überweisungspraxis («EKT als letzte Wiese») steht oftmals nicht im Einklang mit einer leitlinienorientierten Behandlung der therapieresistenten Depression. So empfiehlt beispielsweise die S3-Leitlinie Unipolare Depression, die EKT bei schweren, therapieresistenten depressiven Episoden als Behandlungsalternative in Betracht zu ziehen. Demnach kann die EKT auch zur Erhaltungstherapie eingesetzt werden bei Patient*innen, die während einer 
Krankheitsepisode auf EKT angesprochen haben, auf eine andere leitliniengerechte antidepressive Therapie nicht angesprochen haben, psychotische Merkmale aufweisen oder eine entsprechende Präferenz haben.

Untersuchungen unter anderem mittels bildgebender Verfahren haben Kenntnisse zu den neurophysiologischen und neurochemischen Substraten der Wirkung der EKT geliefert bzw. werden weitere liefern. Anwendungsstudien werden es ermöglichen, Applikationsmethoden zu entwickeln, die mit geringeren neuropsychologischen Nebenwirkungen verknüpft sind.

Ein zentrales Anliegen besteht jedoch in der weiterhin notwendigen Enttabuisierung der EKT: «Die immer wieder gezielt in die Öffentlichkeit getragene Darstellung der Elektrokrampftherapie als veraltete, überholte oder gar inhumane und grausame Behandlungsmethode ist falsch und beruht weitgehend auf einer mangelhaften Information» (Stellungnahme der Deutschen Bundesärztekammer; Folkerts et al., 2003). Die skeptische Einschätzung der EKT entspricht nicht dem Forschungsstand, vielmehr sollte die EKT im Rahmen einer umfassenden Therapieplanung als eine mögliche Behandlungsoption angeboten werden - und nicht nur als «Ultima ratio» (Tölle, 2008). Trotz der grundsätzlichen Unterschiede der theoretischen Konzeptualisierung der jeweiligen psychiatrischen Erkrankungen besteht kein grundsätzlicher Widerspruch zwischen einer indizierten Behandlung mittels EKT und der Durchführung einer Psychotherapie: Im Gegenteil, oftmals ermöglicht erst die EKT die Überwindung depressiver Blockaden, die Auseinandersetzung mit biografisch relevanten Konfliktthemen, dysfunktionalen Bewältigungsmechanismen, traumatischen Erfahrungen und nicht zuletzt auch dem «existenziellen Thema der verlorenen Lebenszeit» infolge einer therapieresistenten Depression.

\section{Literatur}

Abbott, C.C., Lemke, N.T., Gopal, S. et al. (2013). Electroconvulsive therapy response in major depressive disorder: a pilot functional network connectivity resting state FMRI investigation. Front Psychiat, 4(10), 1-9.

Abrams, R. (1992). Electroconvulsive Therapy. New York: Oxford UP. Abrams, R. \& Sukartz, C.M. (1985). ECT and Prolactin release: effects of stimulus parameters. Convuls Ther, 1, 115-119.

APA (1994). Diagnostic and statistical manual of mental disorders (DSM-IV). Washington, D.C.: APA.

Aperia, B., Thoren, M. \& Wetterberg, L. (1985). Prolactin and thyrotropin in serum during electroconvulsive therapy in patients with major depressive illness. Acta Psychiatr Scand, 72, 302-308.

Bahji, A., Hawken, E.R., Sepehry, A.A. et al. (2019). ECT beyond unipolar major depression: systematic review and meta-analysis of electroconvulsive therapy in bipolar depression. Acta Psychiatr Scand, 139(3), 214-226.

Baldessarini, R.J., Tondo, L. \& Hennen, J. (2003). Lithium treatment and suicide risk in major affective disorders: update and new findings. J Clin Psychiat, 64(Suppl. 5), 44-52.

Baldinger, P., Lotan, A., Frey, R. et al. (2014). Neurotransmitters and Electroconvulsive Therapy. J ECT, 30, 116-121.

Benbow, S.M. \& Crentsil, J. (2004). Subjective experience of electroconvulsive therapy. Psychiat Bull, 28, 289-291.
Bilgen, A.E., Bozkurt Zincir, S., Zincir, S. et al. (2014). Effects of electroconvulsive therapy on serum levels of brain-derived neurotrophic factor and nerve growth factor in treatment resistant major depression. Brain Res Bull, 104, 82-87.

Bøg, F.K., Jørgensen, M.B., Andersen, Z.J. \& Osler, M. (2018). Electroconvulsive therapy and subsequent epilepsy in patients with affective disorders: A register-based Danish cohort study. Brain Stimul, 11(2), 411-415.

Bolwig, T.G. \& Madsen, T.M. (2007). Electroconvulsive therapy in melancholia: the role of hippocampal neurogenesis. Acta Psychiatr Scand, (Suppl. 433), 130-135.

Brakemeier, E.L., Merkl, A., Wilbertz, G. et al. (2014). Cognitive-behavioral therapy as continuation treatment to sustain response after electroconvulsive therapy in depression: a randomized controlled trial. Biol Psychiat, 76(3), 194-202.

Brown, E.D., Lee, H., Scott, D. \& Cummings, G.G (2014). Efficacy of continuation/maintenance electroconvulsive therapy for the prevention of recurrence of a major depressive episode in adults with unipolar depression: a systematic review. J ECT, 30(3), 195-202.

Chu, C.W., Chien, W.C., Chung, C.H. et al. (2018). Electroconvulsive Therapy and Risk of Dementia - A Nationwide Cohort Study in Taiwan. Front Psychiat, 9, 397.

Conca, A., Prapotnik, M., Di Pauli, J. \& Wild, B. (2005). Psychotherapie und Elektrokonvulsionstherapie. Widerspruch oder Ergänzung. Nervenheilkunde, 24, 729-735.

DGBS \& DGPPN (2020). Diagnostik und Therapie bipolarer Störungen. In S3-Leitlinie, Langvers. 2.1 (Update v. Feb. 2019, letzte Anpassung Mai 2020).

DGPPN (2017). S3-Leitlinie/Nationale Versorgungs-Leitlinie «Unipolare Depression», Kurzfassung. 2. Aufl., Vers. 1. http://www. versorgungsleitlinien.de

Dogan, S., Aksoy, I. \& Kupka, R.W. (2016). Aortaruptuur als fatale complicatie bij elektroconvulsietherapie [Fatal aortarupture following electroconvulsive therapy]. Tijdschr Psychiatr, 58(2), 150-153.

Fernie, G., Bennett, D.M., Currie, J. et al. (2014). Detecting objective and subjective cognitive effects of electroconvulsive therapy: intensity, duration and test utility in a large clinical sample. Psychol Med, 44(14), 2985-2994.

Fink, M. \& Nemeroff, C.B. (1989). A neuroendocrine view of ECT. Convuls Ther, 5, 296-304.

Florkowski, C.M., Crozier, I.G., Nightingale, S. et al. (1996). Plasma cortisol, PRL, ACTH, AVP and corticotrophin releasing hormone responses to direct current cardioversion and electroconvulsive therapy. Clin Endocrinol, 44, 163-168.

Folkerts, H., Remschmidt, H., Saß, H. et al. (2003). Stellungnahme zur Elektrokrampftherapie (EKT) als psychiatrische Behandlungsmaßnahme. Dtsch Arztebl, 100(8), A-504-506.

Fraser, L.M., O'Carroll, R.E. \& Ebmeier, K.P. (2008). The effect of electroconvulsive therapy on autobiographical memory: a systematic review. J ECT, 24(1), 10-17.

Gbyl, K. \& Videbech, P. (2018). Electroconvulsive therapy increases brain volume in major depression: a systematic review and meta-analysis. Acta Psychiatr Scand, 138(3), 180-195.

Grager, B. \& Di Pauli, J. (2013). Indikationen und Wirksamkeit der EKT. In M. Grözinger, A. Conca, T. Nickl-Jockschat \& J. Di Pauli (Hrsg.), Elektrokonvulsionstherapie kompakt: Für Zuweiser und Anwender (S. 73-80). Berlin, Heidelberg: Springer.

Grözinger, M., Conca, A., Nickl-Jockschat, T. \& Di Pauli, J. (2013). Vorwort. In dies. (Hrsg.), Elektrokonvulsionstherapie kompakt: Für Zuweiser und Anwender (S. V-VI). Berlin, Heidelberg: Springer.

Gugger, J.J. \& Dunn, L.E. (2019). Subarachnoid Hemorrhage in the Setting of Electroconvulsive Therapy in a Patient with an Unsecured Cerebral Aneurysm: A Case Report and Review of the Literature. J ECT, 35(3), 212-214.

Haq, A.U., Sitzmann, A.F., Goldman, M.L. et al. (2015). Response of depression to electroconvulsive therapy: a meta-analysis of clinical predictors. J Clin Psychiat, 76(10), 1374-1384. 
Himmighoffen, H. (2009). Einzelpsychotherapie: Die Rolle der medikamentösen Behandlung. In H. Böker, D. Hell \& D. Teichman (Hrsg.), Teilstationäre Behandlung von Depressionen, Angst- und Zwangsstörungen: Tagesklinik für Affektkranke (S. 51-54). Stuttgart, Schattauer.

Holsboer-Trachsler, E., Hättenschwiler, J., Beck, J. et al. (2016a). Die Akutbehandlung depressiver Episoden. Die somatische Behandlung der unipolaren depressiven Störungen: Update 2016, Teil 1. Swiss Med Forum, 16(35), 716-724.

Holsboer-Trachsler, E., Hättenschwiler, J., Beck, J. et al. (2016b). Erhaltungstherapie und Rezidivprophylaxe unipolarer depressiver Störungen. Die somatische Behandlung der unipolaren depressiven Störungen: Update 2016, Teil 1. Swiss Med Forum, 16(36), 739-743.

Ishihara, K. \& Sasa, M. (1999). Mechanism underlying the therapeutic effects of electroconvulsive therapy (ECT) on depression. Jpn J Pharmacol, 80, 185-189.

Kalisova, L., Kubinova, M., Michalec, J. et al. (2018). Cognitive functioning in patients treated with electroconvulsive therapy. Neuropsychiatr Dis Treat, 14, 3025-3031.

Kanai, T., Takeuchi, H., Furukawa, T.A. et al. (2003). Time to recurrence after recovery from major depressive episodes and its predictors. Psychol Med, 33, 839-845.

Kay, J. (2007). Psychotherapy and medication. In G.O. Gabbard, J.S. Beck \& J. Holmes (Hrsg.), Oxford Textbook of Psychotherapy (S. 463-475). New York: Oxford UP.

Keller, M.B., Lavori, P.W., Endicott, J. et al. (1983). «Double depression»: Two-year follow-up. Am J Psychiat, 140(6), 689-694.

Kellner, C.H. (2019). Handbook of ECT. A Guide to Electroconvulsive Therapy. Cambridge, New York: UP.

Kellner, C.H., Fink, M., Knapp, R. et al. (2005). Relief of expressed suicidal intent by ECT: a consortium for research in ECT study. Am J Psychiat, 162(5), 977-982.

Kellner, C.H., Knapp, R.G., Petrides, G. et al. (2006). Continuation electroconvulsive therapy vs pharmacotherapy for relapse prevention in major depression: a multisite study from the Consortium for Research in Electroconvulsive Therapy (CORE). Arch Gen Psychiat, 63(12), 1337-1344.

Kelly, C.B. \& Cooper, S.J. (1997). Plasma noradrenaline response to electroconvulsive therapy in depressive illness. Br J Psychiat, 171, 182-186.

Kocsis, J.H. (2000). New strategies for treating chronic depression. J Clin Psychiat, 61(Suppl. 11), 42-45.

König, K. (1999). Psychoanalyse in der psychiatrischen Arbeit - eine Einführung. Bonn: Psychiatrie-Verlag.

Lally, J., Tully, J., Robertson, D. et al. (2016). Augmentation of clozapine with electroconvulsive therapy in treatment resistant schizophrenia: A systematic review and meta-analysis. Schizophr Res, 171(1-3), 215-224.

Lamont, S.R., Stanwell, B.J., Hill, R. et al. (2005). Ketamine pre-treatment dissociates the effects of electroconvulsive stimulation on mossy fibre sprouting and cellular proliferation in the dentate gyrus. Brain Res, 1053(1-2), 27-32.

Lauber, C., Nordt, C., Falcato, L. \& Rossler, W. (2005). Can a seizure help? The public's attitude toward electroconvulsive therapy. Psychiat Res, 134(2), 205-209.

Luccarelli, J., McCoy, T.H. Jr., Seiner, S.J. \& Henry, M.E. (2020). Maintenance ECT is associated with sustained improvement in depression symptoms without adverse cognitive effects in a retrospective cohort of 100 patients each receiving 50 or more ECT treatments. J Affect Disord, 271, 109-114.

Luchini, F., Medda, P., Mariani, M.G. et al. (2015). Electroconvulsive therapy in catatonic patients: Efficacy and predictors of response. World J Psychiatry, 5(2), 182-192.

Madsen, T.M., Treschow, A., Bengzon, J. et al. (2000). Increased neurogenesis in a model of electroconvulsive therapy. Biol Psychiat, 47(12), 1043-1049.

McIntyre, R.S., Filteau, M.J., Martin, L. et al. (2014). Treatment-resistant depression: definitions, review of the evidence, and algorithmic approach. J Affect Disord, 156, 1-7.
Meister, R., Jansen, A., Berger, M. et al. (2018). Psychotherapie depressiver Störungen: Verfahren, Evidenz und Perspektiven [Psychotherapy of depressive disorders: Procedures, evidence and perspectives]. Nervenarzt, 89(3), 241-251.

Michael, N., Erfurth, A., Ohrmann, P. et al. (2003a). Neurotrophic effects of electroconvulsive therapy: a proton magnetic resonance study of the left amygdalar region in patients with treatment-resistant depression. Neuropsychopharmacol, 28(4), 720-725.

Michael, N., Erfurth, A., Ohrmann, P. et al. (2003b). Metabolic changes within the left dorsolateral prefrontal cortex occurring with electroconvulsive therapy in patients with treatment resistant unipolar depression. Psychol Med, 33(7), 1277-1284.

Milev, R.V., Giacobbe, P., Kennedy, S. et al. (2016). Canadian Network for Mood and Anxiety Treatments (CANMAT) 2016 Clinical Guidelines for the Management of Adults with Major Depressive Disorder: Section 4. Neurostimulation Treatments. Can J Psychiat, 61(9), 561-575.

Mueller, P.S., Albin, S.M., Barnes, R.D. \& Rasmussen, K.G. Jr. (2009). Safety of electroconvulsive therapy in patients with unrepaired abdominal aortic aneurysm: report of 8 patients. J ECT, $25(3), 165-169$.

Nuninga, J.O., Claessens, T.F.I., Somers, M. et al. (2018). Immediate and long-term effects of bilateral electroconvulsive therapy on cognitive functioning in patients with a depressive disorder. $J$ Affect Disord, 238, 659-665.

Nuninga, J.O., Mandl, R.C.W., Boks, M.P. et al. (2019). Volume increase in the dentate gyrus after electroconvulsive therapy in depressed patients as measured with 7T. Mol Psychiat. https://doi. org/10.1038/s41380-019-0392-6

Obbels, J., Verwijk, E., Vansteelandt, K. et al. (2018). Long-term neurocognitive functioning after electroconvulsive therapy in patients with late-life depression. Acta Psychiatr Scand, 138(3), 223-231.

Okamoto, T., Yoshimura, R., Ikenouchi-Sugita, A. et al. (2008). Efficacy of electroconvulsive therapy is associated with changing blood levels of homovanillic acid and brain-derived neurotrophic factor (BDNF) in refractory depressed patients: a pilot study. Prog Neuropsychopharmacol Biol Psychiat, 32, 1185-1190.

Olesen, M.V., Wörtwein, G., Folke, J. \& Pakkenberg, B. (2017). Electroconvulsive stimulation results in long-term survival of newly generated hippocampal neurons in rats. Hippocampus, 27(1), 52-60.

Osler, M., Rozing, M.P., Christensen, G.T. et al. (2018). Electroconvulsive therapy and risk of dementia in patients with affective disorders: a cohort study. Lancet Psychiat, 5(4), 348-356.

Otabe, H., Nibuya, M., Shimazaki, K. et al. (2014). Electroconvulsive seizures enhance autophagy signaling in rat hippocampus. Prog Neuropsychopharmacol Biol Psychiat, 3(50), 37-43.

Pagnin, D., de Queiroz, V., Pini, S. \& Cassano, G.B. (2004). Efficacy of ECT in depression: a meta-analytic review. J ECT, 20(1), 13-20.

Pandarakalam, J.P. (2018). Challenges of Treatment-resistant Depression. Psychiatr Danub, 30(3), 273-284.

Perrin, J.S., Merz, S., Bennett, D.M. et al. (2012). Electroconvulsive therapy reduces frontal cortical connectivity in severe depressive disorder. Proc Natl Acad Sci USA, 109, 5464-5468.

Perugi, G., Medda, P., Toni, C. et al. (2017). The Role of Electroconvulsive Therapy (ECT) in Bipolar Disorder: Effectiveness in 522 Patients with Bipolar Depression, Mixed-state, Mania and Catatonic Features. Curr Neuropharmacol, 15(3), 359-371.

Petrides, G., Malur, C., Braga, R.J. et al. (2015). Electroconvulsive therapy augmentation in clozapine-resistant schizophrenia: a prospective, randomized study. Am J Psychiat, 172(1), 52-58.

Pfaff, M., Seidl, A., Angst, K. et al. (2013). Elektrokonvulsionstherapie als Ultima Ratio in der Behandlung der Depression? [Electroconvulsive therapy as a «last resort» in the treatment of depression?] Psychiatr Prax, 40(7), 385-390.

Pfleiderer, B., Michael, N., Erfurth, A. et al. (2003). Effective electroconvulsive therapy reverses glutamate/glutamine deficit in the left anterior cingulum of unipolar depressed patients. Psychiat Res, 122(3), 185-192. 
Piccinni, A., Del Debbio, A., Medda, P. et al. (2009). Plasma Brain-Derived Neurotrophic Factor in treatment-resistant depressed patients receiving electroconvulsive therapy. Eur Neuropsychopharmacol, 19(5), 349-355.

Read, J. \& Arnold, C. (2017). Is Electroconvulsive Therapy for Depression More Effective Than Placebo? A Systematic Review of Studies Since 2009. EHPP, 19(1), 5-23.

Read, J. \& Bentall, R. (2010). The effectiveness of electroconvulsive therapy: A literature review. Epidemiol Psychiatr Sci, 19(4), 333-347.

Reinke, V., Bertram, L. \& Grözinger, M. (2013). Geschichte der Elektrokonvulsionstherapie. In M. Grözinger, A. Conca, T. NicklJockschat \& J. Di Pauli (Hrsg.), Elektrokonvulsionstherapie kompakt: Für Zuweiser und Anwender (S. 3-14). Berlin, Heidelberg: Springer.

Rocha, R.B., Dondossola, E.R., Grande, A.J. et al. (2016). Increased BDNF levels after electroconvulsive therapy in patients with major depressive disorder: A meta-analysis study. I Psychiatr Res, 83, 47-53.

Rozing, M.P., Jorgensen, M.B. \& Osler, M. (2019): Electroconvulsive therapy and later stroke in patients with affective disorders. $\mathrm{Br} J$ Psychiat, 214(3), 168-170.

Sackeim, H.A. (2017). Modern Electroconvulsive Therapy: Vastly Improved yet Greatly Underused. JAMA, 74(8), 779-780.

Sackeim, H.A., Haskett, R.F., Mulsant, B.H. et al. (2001). Continuation Pharmacotherapy in the Prevention of Relapse Following Electroconvulsive Therapy - A Randomized Controlled Trial. JAMA, 285(10), 1299-1307.

Sanacora, G., Mason, G.F., Rothman, D.L. et al. (2003). Increased cortical GABA concentrations in depressed patients receiving ECT. Am J Psychiat, 160(3), 577-579.

Sartorius, A., Demirakca, T., Böhringer, A. et al. (2019). Electroconvulsive therapy induced gray matter increase is not necessarily correlated with clinical data in depressed patients. Brain Stimul, 12(2), 335-343.

Sartorius, A., Pycha, R., Grözinger, M. \& Conca, A. (2013). Praktische Durchführung der EKT. In M. Grözinger, A. Conca, T. Nickl-Jockschat \& J. Di Pauli (Hrsg.), Elektrokonvulsionstherapie kompakt: Für Zuweiser und Anwender (S. 109-125). Berlin, Heidelberg: Springer.

Schloesser, R.J., Orvoen, S., Jimenez, D.V. et al. (2015). Antidepressant-like Effects of Electroconvulsive Seizures Require Adult Neurogenesis in a Neuroendocrine Model of Depression. Brain Stimul, $8(5), 862-867$.

Schulz-Du Bois, A.C. \& Conca, A. (2013). EKT: Komedikation und begleitende Therapien. In M. Grözinger, A. Conca, T. Nickl-Jockschat \& J. Di Pauli (Hrsg.), Elektrokonvulsionstherapie kompakt: Für Zuweiser und Anwender (S. 155-171). Berlin, Heidelberg: Springer.

Scott, A.I.F., Lawrence, J., Whalley, L.J. \& Legros, J.L. (1989). Treatment Outcome, Seizure Duration, and the Neurophysin Response to ECT. Biol Psychiat, 25, 585-597.

Semkovska, M. \& McLoughlin, D.M. (2010). Objective Cognitive Performance Associated with Electroconvulsive Therapy for Depression: A Systematic Review and Meta-Analysis. Biol Psychiat, 68(6), 568-577.

Smith, J.S., Roopa, R., Sagar, B.K. et al. (2014). Images in electroconvulsive therapy: ECS dose-dependently increases cell proliferation in the subgranular region of the rat hippocampus. J ECT, 30(3), $193 \mathrm{f}$.

Svensson, M., Grahm, M., Ekstrand, J. et al. (2016). Effect of electroconvulsive seizures on cognitive flexibility. Hippocampus, 26(7), 899-910.

Takamiya, A., Chung, J.K., Liang, K.C. et al. (2018). Effect of electroconvulsive therapy on hippocampal and amygdala volumes: systematic review and meta-analysis. Br J Psychiat, 212(1), 19-26.

Takamiya, A., Plitman, E., Chung, J.K. et al. (2019). Acute and long-term effects of electroconvulsive therapy on human dentate gyrus. Neuropsychopharmacol. https://doi.org/10.1038/s41386019-0312-0
Tendolkar, I., van Beek, M., van Oostrom, I. et al. (2013). Electroconvulsive therapy increases hippocampal and amygdala volume in therapy refractory depression: A longitudinal pilot study. Psychiat Res, 214(3), 197-203

Thase, M.E., Greenhouse, J.B., Frank, E. et al. (1997). Treatment of Major Depression with Psychotherapy or Psychotherapy-Pharmacotherapy Combinations. Arch Gen Psychiat, 54, 1009-1015.

Tölle, R. (2008). Nicht die Elektrokrampftherapie, sondern ihre Gegner stehen in der Kritik [Not electroconvulsive therapy, but its opponents are in the firing line]. Nervenarzt, 79(11), 1310-1312.

Torring, N., Sanghani, S.N., Petrides, G. et al. (2017). The mortality rate of electroconvulsive therapy: a systematic review and pooled analysis. Acta Psychiatr Scand, 135(5), 388-397.

UK ECT Review Group (2003). Efficacy and safety of electroconvulsive therapy in depressive disorders: a systematic review and meta-analysis. Lancet 361, 799-808.

Whalley, L.J., Eagles, J.M., Bowler, G.M. et al. (1987). Selective effects of ECT on hypothalamic-pituitary activity. Psychol Med, 17, 319-328.

\section{The importance of electroconvulsive therapy in the multimodal treatment of depressive disorders}

Abstract: Electroconvulsive therapy (ECT) is part of the multimodal treatment concept in the treatment of depressive disorders in particular. Therapy-resistant depression is regarded as a major indication. After decades of experience, ECT continues to prove to be a very effective treatment method that is safe and has relatively few side effects due to current standards and technology. Its prejudiced and negative perception and evaluation is therefore surprising. The very frequent assessment of ECT as a last resort means that in many cases it is only used at a very late stage in the course of the disease - despite recommendations to the contrary, for example the S3 Guideline Unipolar Depression of the DGPPN. The indication range for ECT is clearly defined and there are only few absolute contraindications; a combination of ECT with other treatment procedures is also possible and sensible. The central concern of this article is the necessary removal of taboos: ECT should be offered as a possible treatment option as part of a comprehensive therapy plan. In many cases, it is the only way to overcome depressive blockages, to deal with biographically relevant conflict issues, dysfunctional coping mechanisms, traumatic experiences, and also the «existential issue of lost lifetime» as a result of therapy-resistant depression.

Key words: electroconvulsive therapy (ECT), therapy-resistant depression, psychotherapy, multimodal treatment

\section{L'importanza della terapia elettroconvulsivante (TEC) nel trattamento multimodale dei disturbi depressivi}

Riassunto: La terapia elettroconvulsivante (TEC) è parte del piano di trattamento con approccio multimodale nella cura delle patologie soprattutto di natura depressiva. La depressione resistente alle terapie va considerata come un'indicazione chiave. Dopo decenni di esperienza la TEC si è rivelata essere un metodo di trattamento ancora molto efficace e, a fronte degli standard e delle tecnologie attuali, più sicuro e con effetti collaterali relativamente contenuti. Perciò sorprende che tale metodo sia ancora percepito e valutato in 
modo così pregiudizievole e negativo. Anche la posizione molto frequente secondo cui la TEC debba essere utilizzata in ultima ratio comporta che il ricorso a tale metodo nel trattamento avvenga solo in uno stadio molto avanzato della patologia, nonostante le diverse raccomandazioni, come la linea guida nazionale S3 per il trattamento della depressione unipolare della DGPPN, Deutsche Gesellschaft für Psychiatrie und Psychotherapie, Psychosomatik und Nervenheilkunde (Società tedesca di psichiatria e psicoterapia, psicosomatica e neurologia). Le indicazioni per il ricorso alla TEC sono chiaramente definite e sussistono esigue controindicazioni che possano escluderne in modo assoluto l'impiego. Inoltre è possibile e sensato combinare la TEC con altre metodologie di trattamento. La questione centrale del presente articolo è rappresentata da un tentativo di smantellamento dei tabù legati a tale metodo: la TEC dovrebbe essere proposta come possibile opzione nell'ambito di un trattamento terapico a $360^{\circ}$. Spesso è proprio il ricorso a tale metodo che rende possibile il superamento dei blocchi causati dalla depressione, il confronto con tematiche conflittuali rilevanti nella biografia del paziente, i meccanismi per riuscire ad affrontare le situazioni più problematiche, le esperienze traumatiche e anche il «tema esistenziale del tempo ormai trascorso e perso" a causa di una patologia depressiva resistente alle terapie.

Parole chiave: terapia elettroconvulsivante (TEC), depressione resistente alle terapie, psicoterapia, trattamento multimodale

\section{Die Autoren}

Holger Himmighoffen, Dr. med., ist Facharzt für Psychiatrie und Psychotherapie FMH, Psychoanalytiker in eigener Praxis in Zürich (Zentrum für Psychiatrie, Psychotherapie und Psychoanalyse) und leitender Oberarzt im Ambulatorium für Elektrokonvulsionstherapie der Klinik für Psychiatrie, Psychotherapie und Psychosomatik der Psychiatrischen Universitätsklinik Zürich.

Heinz Böker, Prof. Dr. med., ist Facharzt für Psychiatrie und Psychotherapie FMH, Kinder- und Jugendpsychiatrie und Psychosomatische Medizin, Psychoanalytiker im Zentrum für Psychiatrie, Psychotherapie und Psychoanalyse, Titularprofessor für Psychiatrie (em.) an der Universität Zürich und Associate Professor am Zentrum für Psychiatrische Forschung, Psychiatrische Universitätsklinik Zürich. Zuvor war er an der Psychiatrischen Universitätsklinik Zürich tätig und Chefarzt des Zentrums für Depressionen, Angststörungen und Psychotherapie.

\section{Kontakt}

Dr. med. Holger Himmighoffen

Klinik für Psychiatrie, Psychotherapie und Psychosomatik Psychiatrische Universitätsklinik Zürich

Lenggstrasse 31

Postfach 1931

8032 Zürich

Email: holger.himmighoffen@pukzh.ch 